\title{
Levantamento de moscas frugívoras em dois municípios do estado do Acre, Brasil
}

\author{
Tatyane da Silva Azevedo ${ }^{1}$ \\ Adriana da Silva Vasconcelos ${ }^{1}$ \\ Rodrigo Souza Santos ${ }^{2}$ \\ Maria do Socorro Miranda de Sousa ${ }^{3}$ \\ Ricardo Adaime ${ }^{3,4 *}$ \\ ${ }^{1}$ União Educacional do Norte, CEP: 69915-901, Rio Branco - AC, Brasil \\ ${ }^{2}$ Embrapa Acre, CEP: 69900-970, Rio Branco - AC, Brasil \\ ${ }^{3}$ Universidade Federal do Amapá, Programa de Pós-graduação em \\ Biodiversidade Tropical, CEP: 68902-280, Macapá - AP, Brasil \\ ${ }^{4}$ Embrapa Amapá, CEP: 68903-419, Macapá - AP, Brasil \\ * Autor para correspondência \\ ricardo.adaime@embrapa.br
}

Submetido em 01/03/2018

Aceito para publicação em 10/08/2018

\section{Resumo}

Durante os meses de julho de 2016 a setembro de 2017 foram realizadas amostragens de frutos carnosos (38 amostras, 50,93 kg) nos municípios de Rio Branco e Senador Guiomard, estado do Acre, visando ampliar o conhecimento sobre a diversidade de moscas frugívoras, suas plantas hospedeiras e parasitoides associados. Neste trabalho, fizemos o primeiro registro de Anastrepha serpentina (Wiedemann) e do parasitoide Asobara anastrephae (Muesebeck) para o estado do Acre. Além disso, Pouteria caimito (Ruiz \& Pav.) Radlk. (Sapotaceae) constitui novo hospedeiro para Ceratitis capitata na Amazônia brasileira.

Palavras-chave: Anastrepha serpentina; Asobara anastrephae; Distribuição; Hospedeiros

\section{Abstract}

Survey of frugivorous flies in two municipalities in the state of Acre, Brazil. From July 2016 to September 2017, samples of fleshy fruits ( 38 samples, $50.93 \mathrm{~kg}$ ) were collected in the municipalities of Rio Branco and Senador Guiomard, in the state of Acre, Brazil, aiming to expand our knowledge about the diversity of fruit flies, their host plants and associated parasitoids. In this work, we report the first record of Anastrepha serpentina (Wiedemann) and the parasitoid Asobara anastrephae (Muesebeck) in Acre. In addition, Pouteria caimito (Ruiz \& Pav.) Radlk. (Sapotaceae) was found to be a new host for Ceratitis capitata in the Brazilian Amazon.

Key words: Anastrepha serpentina; Asobara anastrephae; Distribution; Host plants 


\section{Introdução}

As moscas-das-frutas (Diptera: Tephritidae) são conhecidas mundialmente como pragas da fruticultura, em virtude dos danos diretos e indiretos que algumas espécies causam à produção (ALUJA, 1994). Por sua vez, moscas frugívoras da família Lonchaeidae têm sido reportadas como invasoras primárias em espécies vegetais comerciais nos últimos anos, especialmente no Brasil (ADAIME et al., 2017a).

Os estudos sobre moscas frugívoras na Amazônia brasileira foram intensificados nos anos recentes, culminando com o avanço do conhecimento sobre a distribuição geográfica das espécies, suas plantas hospedeiras e parasitoides associados. Atualmente, 76 espécies de Anastrepha (Tephritidae), 12 espécies de parasitoides (Braconidae e Figitidae) e 14 espécies de Neosilba (Lonchaeidae) estão assinaladas para a região, o que pode ser facilmente acessado mediante consulta às bases de dados "Anastrepha species and their host in the Brazilian Amazon" (ADAIME et al., 2016), "Fruit flies parasitoids in the Brazilian Amazon" (SOUSA et al., 2016) e "Lonchaeidae from Brazilian Amazon" (PEREIRA; ADAIME, 2016). Também ocorrem na região duas espécies de Tephritidae introduzidas: Ceratitis capitata (Wiedemann), a mosca-do-mediterrâneo, e Bactrocera carambolae Drew \& Hancock, a mosca-da-carambola (SILVA et al., 2011a).

Embora o conhecimento sobre moscas frugívoras tenha aumentado significativamente na Amazônia brasileira, algumas áreas estratégicas da região ainda não foram devidamente estudadas (ADAIME et al., 2017a), como é o caso do estado do Acre, situado no extremo oeste da região Norte do Brasil, composto por 22 municípios distribuídos em mais de 164 mil km², que faz fronteira com a Bolívia e o Peru (PORTAL BRASIL, 2018). Essas regiões fronteiriças na Amazônia são extremamente vulneráveis à introdução de pragas, motivo pelo qual precisam ser estudadas (LEMOS et al., 2011).

A fruticultura no Acre caracteriza-se pela agricultura de base familiar, sendo praticada em pequenas áreas e com utilização de baixa tecnologia, sofrendo maior impacto na produção, principalmente ocasionado pelos problemas fitossanitários (PEREIRA et al., 2011). Os primeiros registros de moscas frugívoras no Acre foram realizados por Thomazini et al. (2003), seguido de outros poucos trabalhos pontuais (THOMAZINI et al., 2006; THOMAZINI; ALBUQUERQUE, 2009; PEREIRA et al., 2010; STRIKIS et al., 2011; ADAIME et al., 2017b). Estão reportadas apenas sete espécies de Tephritidae [Anastrepha coronilli Carrejo \& González, Anastrepha distincta Greene, Anastrepha leptozona Hendel, Anastrepha obliqua (Macquart), Anastrepha striata Schiner, Anastrepha tumida Stone e Ceratitis capitata (Wiedemann)], duas de Lonchaeidae [Neosilba glaberrima (Wiedemann) e Neosilba zadolicha McAlpine \& Steyskal], além de três espécies de parasitoides [Doryctobracon areolatus (Szépligeti), Opius bellus Gahan e Utetes anastrephae (Viereck) (Hymenoptera: Braconidae)] (ADAIME et al., 2016; PEREIRA; ADAIME, 2016; SOUSA et al., 2016; ADAIME et al., 2017b).

Considerando o escasso conhecimento sobre a diversidade, hospedeiros e parasitoides de moscas frugívoras no estado do Acre, é imprescindível o aprofundamento dos estudos sobre esses insetos (PEREIRA et al., 2011). O objetivo deste trabalho foi realizar um levantamento de moscas frugívoras e suas plantas hospedeiras nos municípios de Rio Branco e Senador Guiomard, estado do Acre.

\section{Material e Métodos}

Foram realizadas coletas de frutos no período de julho de 2016 a setembro de 2017, em quintais residenciais das áreas urbanas dos municípios de Rio Branco e Senador Guiomard, estado do Acre. As datas de coleta foram dependentes da disponibilidade de plantas em frutificação. A amostragem foi realizada ao acaso, coletando-se frutos íntegros recém-caídos ao solo e/ou diretamente nas plantas amostradas. Todos os pontos de coleta tiveram suas coordenadas geográficas registradas por GPS. As amostras, compostas por vários frutos agrupados, foram acondicionadas em recipientes plásticos, envoltos com saco de organza, devidamente identificadas, e posteriormente transportadas para o 
Laboratório de Entomologia da Embrapa Acre, em Rio Branco.

No laboratório, foram seguidos os procedimentos preconizados por Silva et al. (2011b). Os frutos foram contados, pesados e dispostos em bandejas plásticas sobre uma fina camada de areia umedecida. As bandejas foram cobertas com organza presa por liga elástica. O material foi examinado a cada três dias e os pupários encontrados foram removidos e transferidos para frascos plásticos transparentes, contendo uma fina camada de vermiculita umedecida. Os frascos foram cobertos com organza e tampa vazada, sendo inspecionados diariamente. A umidade nas bandejas e nos potes era mantida pela reposição de água, com auxílio de uma pisseta. As moscas-das-frutas e os parasitoides que emergiram foram armazenados em frascos de vidro contendo etanol $70 \%$, para posterior identificação.

Os exemplares de Anastrepha foram identificados com o auxílio da chave dicotômica ilustrada de Zucchi et al. (2011). Para a identificação dos parasitoides (Braconidae) foram utilizados os trabalhos de Canal e Zucchi (2000) e Marinho et al. (2011). Espécimes de Neosilba foram identificados pelo Dr. Pedro Carlos Strikis, de acordo com o trabalho de McAlpine e Steyskal (1982).

Foi calculado o índice de infestação das amostras, pela razão entre o número de pupários obtidos na amostra e o seu respectivo peso (em quilogramas).

\section{Resultados}

Foram coletadas 38 amostras de frutos $(50,93 \mathrm{~kg})$, de 21 espécies vegetais pertencentes a 12 famílias (Tabelas 1 e 2). Houve infestação por moscas frugívoras em 12 amostras $(31,6 \%)$, pertencentes a seis espécies vegetais de quatro famílias (Tabelas 1 e 2).

Foram obtidos 449 pupários, de onde emergiram espécimes de Anastrepha Schiner (quatro espécies), Neosilba McAlpine (duas espécies), C. capitata e parasitoides (uma espécie).

\section{Rio Branco}

Foram coletadas 26 amostras de frutos $(31,04 \mathrm{~kg})$ de 17 espécies vegetais pertencentes a 11 famílias. Ocorreu infestação por moscas frugívoras em sete amostras (quatro espécies vegetais pertencentes a quatro famílias), de onde foram obtidos 220 pupários (Tabela 1).

Foram registradas duas espécies de Tephritidae (A. obliqua e A. striata) e duas de Lonchaeidae (N. glaberrima e N. zadolicha). Em frutos de Spondias mombin L. (Anacardiaceae), infestados por A. obliqua, foram obtidos dois exemplares do parasitoide Asobara anastrephae (Muesebeck) (Hymenoptera: Braconidae) (Tabela 1).

\section{Senador Guiomard}

Foram coletadas 12 amostras de frutos $(19,89 \mathrm{~kg})$ de nove espécies vegetais pertencentes a seis famílias. Foi registrada infestação por moscas frugívoras em cinco amostras (três espécies vegetais pertencentes a duas famílias), de onde foram obtidos 229 pupários (Tabela 2).

Foram registradas cinco espécies de Tephritidae [A. leptozona, A. obliqua, Anastrepha serpentina (Wiedemann), A. striata e C. capitata] e duas de Lonchaeidae (N. glaberrima e N. zadolicha) (Tabela 2). 
TABELA 1: Moscas frugívoras em diversas plantas hospedeiras no município de Rio Branco, estado do Acre. Julho de 2016 a agosto de 2017.

\begin{tabular}{|c|c|c|c|c|c|c|}
\hline Data & Coordenadas Geográficas & Plantas hospedeiras & $\begin{array}{l}\text { Peso } \\
(k g)\end{array}$ & Pupários & $\begin{array}{l}\text { Infestação } \\
\text { (Pupários/kg) }\end{array}$ & Espécies \\
\hline 07/jul/16 & $10^{\circ} 01^{\prime} 50.0^{\prime \prime} \mathrm{S} ; 67^{\circ} 41^{\prime} 09.5^{\prime \prime} \mathrm{W}$ & Citrus sinensis & 6,16 & - & - & - \\
\hline 12/jul/16 & $10^{\circ} 01^{\prime} 43.7^{\prime \prime} \mathrm{S} ; 67^{\circ} 41^{\prime} 05.0^{\prime \prime} \mathrm{W}$ & Coffea canephora & 0,07 & - & - & - \\
\hline 28/jul/16 & $10^{\circ} 00^{\prime} 30.1^{\prime \prime} \mathrm{S} ; 67^{\circ} 47^{\prime} 27.0^{\prime \prime} \mathrm{W}$ & Averrhoa carambola & 1,27 & - & - & - \\
\hline 29/jul/16 & $10^{\circ} 07^{\prime} 58.0^{\prime \prime} \mathrm{S} ; 67^{\circ} 50^{\prime} 39.2^{\prime \prime} \mathrm{W}$ & Manihot sculenta & 0,24 & - & - & - \\
\hline $01 /$ set $/ 16$ & $10^{\circ} 00^{\prime} 21.2^{\prime \prime} \mathrm{S} ; 67^{\circ} 83^{\prime} 02.3^{\prime \prime} \mathrm{W}$ & Syzygium jambos & 0,79 & - & - & - \\
\hline $02 /$ set $/ 16$ & $10^{\circ} 00^{\prime} 48.8^{\prime \prime} \mathrm{S} ; 67^{\circ} 42^{\prime} 14.1^{\prime \prime} \mathrm{W}$ & Anacardium occidentale & 1,07 & - & - & - \\
\hline $05 /$ set $/ 16$ & $10^{\circ} 07^{\prime} 58.0^{\prime \prime} \mathrm{S} ; 67^{\circ} 50^{\prime} 39.2^{\prime \prime} \mathrm{W}$ & Annona muricata & 1,38 & - & - & - \\
\hline $28 /$ set $/ 16$ & $10^{\circ} 00^{\prime} 30.1^{\prime \prime} \mathrm{S} ; 67^{\circ} 47^{\prime} 27.0^{\prime \prime} \mathrm{W}$ & Morinda citrifolia & 1,30 & - & - & - \\
\hline 28/out/16 & 0959'12.0”S; 6748’29.2”'W & Averrhoa carambola & 1,33 & - & - & - \\
\hline $23 /$ nov/16 & $10^{\circ} 01^{\prime} 31.2^{\prime \prime} \mathrm{S} ; 67^{\circ} 42^{\prime} 24.1^{\prime \prime} \mathrm{W}$ & Solanum aculeatissimum & 0,05 & - & - & - \\
\hline $24 /$ nov/16 & 1001’34.6”S; 67²4'17.8”W & Syzygium jambolanum & 0,28 & - & - & - \\
\hline 07/dez/16 & $10^{\circ} 00^{\prime} 30.1^{\prime \prime S} ; 67^{\circ} 47^{\prime} 27.0^{\prime \prime} \mathrm{W}$ & Malpighia emarginata & 0,44 & - & - & - \\
\hline $18 / \mathrm{dez} / 16$ & $10^{\circ} 07^{\prime} 58.0^{\prime \prime} \mathrm{S} ; 67^{\circ} 50^{\prime} 39.2^{\prime \prime} \mathrm{W}$ & Bellucia grossularioides & 0,86 & - & - & - \\
\hline 31/jan/17 & $10^{\circ} 01^{\prime} 27.2^{\prime \prime S} ; 67^{\circ} 42^{\prime} 1.70^{\prime \prime} \mathrm{W}$ & Spondias mombin & 1,40 & 88 & 62,9 & $\begin{array}{l}\text { Anastrepha obliqua }(6+) \\
\text { Asobara anastrephae }\left(2{ }^{\top}\right)\end{array}$ \\
\hline $14 / \mathrm{fev} / 17$ & $10^{\circ} 00^{\prime} 30.1^{\prime \prime} \mathrm{S} ; 67^{\circ} 47^{\prime} 27.0^{\prime \prime} \mathrm{W}$ & Psidium guajava & 1,12 & 26 & 23,2 & Anastrepha striata $(7 \bigcirc+7 \hat{+})$ \\
\hline 26/maio/17 & $10^{\circ} 00^{\prime} 11.2^{\prime \prime} \mathrm{S} ; 67^{\circ} 83^{\prime} 16.8^{\prime \prime} \mathrm{W}$ & Averrhoa carambola & 0,54 & - & - & - \\
\hline 26/maio/17 & $10^{\circ} 00^{\prime} 11.2^{\prime \prime} \mathrm{S} ; 67^{\circ} 83^{\prime} 16.8^{\prime \prime} \mathrm{W}$ & Eugenia stipitata & 0,46 & - & - & - \\
\hline 28/maio/17 & $10^{\circ} 00^{\prime} 30.1^{\prime \prime} \mathrm{S} ; 67^{\circ} 47^{\prime} 27.0^{\prime \prime} \mathrm{W}$ & Averrhoa carambola & 1,65 & - & - & - \\
\hline 30/maio/17 & $10^{\circ} 00^{\prime} 11.2^{\prime \prime} \mathrm{S} ; 67^{\circ} 83^{\prime} 16.8^{\prime \prime} \mathrm{W}$ & Averrhoa carambola & 0,57 & - & - & - \\
\hline 31/maio/17 & $10^{\circ} 01^{\prime} 50,0^{\prime \prime} \mathrm{S} ; 67^{\circ} 41^{\prime} 09.5^{\prime \prime} \mathrm{W}$ & Citrus sinensis & 4,29 & 54 & 12,6 & $\begin{array}{c}\text { Neosilba glaberrima }\left(18 \bigcirc^{\Uparrow}\right) \\
\text { Neosilba zadolicha }\left(17 \hat{\jmath}^{\lambda}\right) \\
\text { Neosilba sp. }(15+) \\
\end{array}$ \\
\hline 15/jun/17 & 0957’58.1”S; 6749’00.6”W & Psidium guajava & 0,43 & 33 & 76,7 & Anastrepha striata $\left(12++15{ }^{\Uparrow}\right)$ \\
\hline 09/ago/17 & 0956'51.1”S; 67053'15.1”W & Inga edulis & 0,93 & - & - & - \\
\hline 14/ago/17 & $10^{\circ} 00^{\prime} 46.1^{\prime \prime} \mathrm{S} ; 67^{\circ} 43^{\prime} 53.1^{\prime \prime} \mathrm{W}$ & Psidium guajava & 2,11 & 6 & 2,84 & Anastrepha striata $\left(5++1{ }^{\Uparrow}\right)$ \\
\hline 18/ago/17 & $10^{\circ} 00^{\prime} 48.8^{\prime \prime} \mathrm{S} ; 67^{\circ} 42^{\prime} 14.1^{\prime \prime} \mathrm{W}$ & Anacardium occidentale & 1,80 & 13 & 7,2 & Anastrepha obliqua $\left(2++6 \bigcirc^{\lambda}\right)$ \\
\hline 18/ago/17 & $10^{\circ} 00^{\prime} 48.8^{\prime \prime} \mathrm{S} ; 67^{\circ} 42^{\prime} 14.1^{\prime \prime} \mathrm{W}$ & Citrus reticulata & 0,50 & - & - & - \\
\hline
\end{tabular}


TABELA 2: Moscas frugívoras em diversas plantas hospedeiras no município de Senador Guiomard, estado do Acre. Agosto de 2016 a setembro de 2017.

\begin{tabular}{|c|c|c|c|c|c|c|}
\hline Data & Coordenadas Geográficas & Plantas hospedeiras & $\begin{array}{l}\text { Peso } \\
(\mathrm{kg})\end{array}$ & Pupários & $\begin{array}{l}\text { Infestação } \\
\text { (Pupários/kg) }\end{array}$ & Espécies \\
\hline 02/ago/16 & $10^{\circ} 07^{\prime} 20.5^{\prime \prime} \mathrm{S} ; 67^{\circ} 39^{\prime} 46.4^{\prime \prime} \mathrm{W}$ & Inga edulis & 1,07 & - & - & - \\
\hline 15/ago/16 & $10^{\circ} 00^{\prime} 21.2^{\prime \prime} \mathrm{S} ; 67^{\circ} 83^{\prime} 02.3^{\prime \prime} \mathrm{W}$ & Anacardium occidentale & 1,06 & - & - & - \\
\hline $28 /$ set $/ 16$ & $10^{\circ} 07^{\prime} 34.3^{\prime \prime} \mathrm{S} ; 67^{\circ} 38^{\prime} 47.1^{\prime \prime} \mathrm{W}$ & Mangifera indica & 4,34 & - & - & - \\
\hline $24 / a b r / 17$ & $10^{\circ} 00^{\prime} 30.1^{\prime \prime S} ; 67^{\circ} 38^{\prime} 47.1^{\prime \prime} \mathrm{W}$ & Eugenia stipitata & 1,08 & 104 & 96,3 & $\begin{array}{c}\text { Anastrepha obliqua }(799+7 \hat{\jmath}) \\
\text { Neosilba zadolicha }\left(1 \hat{\delta}^{\Uparrow}\right)\end{array}$ \\
\hline 14/jun/17 & $10^{\circ} 09^{\prime} 11.0^{\prime \prime} \mathrm{S} ; 67^{\circ} 43^{\prime} 42.5^{\prime \prime} \mathrm{W}$ & Pouteria caimito & 1,22 & 1 & 0,82 & Neosilba sp. $(1+$ ) \\
\hline 26/jun/17 & $10^{\circ} 09^{\prime} 11.0^{\prime \prime} \mathrm{S} ; 67^{\circ} 43^{\prime} 42.5^{\prime \prime} \mathrm{W}$ & Pouteria caimito & 4,36 & 71 & 16,3 & $\begin{array}{c}\text { Anastrepha serpentina }(8 \bigcirc) \\
\text { Anastrepha leptozona }(8 \bigcirc) \\
\text { Anastrepha sp. }(24 \hat{\bigcirc}) \\
\text { Ceratitis capitata }\left(2++1{ }^{\lambda}\right) \\
\text { Neosilba glaberrima }(3 \hat{\circ}+1+))\end{array}$ \\
\hline 24/jul/17 & 1007’24.7”S; 67³8'47.1”W & Citrus limetta & 2,03 & - & - & - \\
\hline 24/jul/17 & $10^{\circ} 07^{\prime} 24.7^{\prime \prime} \mathrm{S} ; 67^{\circ} 38^{\prime} 47.1^{\prime \prime} \mathrm{W}$ & Citrus aurantifolia & 2,28 & - & - & - \\
\hline 09/ago/17 & $10^{\circ} 07^{\prime} 24.7^{\prime \prime} \mathrm{S} ; 67^{\circ} 38^{\prime} 47.1^{\prime \prime} \mathrm{W}$ & Psidium guajava & 0,42 & 42 & 100 & Anastrepha striata $\left(13 \uparrow+5{ }^{\lambda}\right)$ \\
\hline 09/ago/17 & $10^{\circ} 07^{\prime} 24.7^{\prime \prime} \mathrm{S} ; 67^{\circ} 38^{\prime} 47.1^{\prime \prime} \mathrm{W}$ & Annona muricata & 0,95 & - & - & - \\
\hline $03 /$ set $/ 17$ & $10^{\circ} 07^{\prime} 24.7^{\prime \prime} \mathrm{S} ; 67^{\circ} 38^{\prime} 47.1^{\prime \prime} \mathrm{W}$ & Anacardium occidentale & 0,58 & - & - & - \\
\hline $03 /$ set/17 & $10^{\circ} 14^{\prime} 55.1^{\prime \prime} \mathrm{S} ; 67^{\circ} 73^{\prime} 20.1^{\prime \prime} \mathrm{W}$ & Pouteria caimito & 0,50 & 11 & 22 & $\begin{array}{c}\text { Anastrepha leptozona }\left(1++2{ }^{\top}\right) \\
\text { Ceratitis capitata }(7+9)\end{array}$ \\
\hline
\end{tabular}

\section{Discussão}

Neste trabalho, é feito o primeiro registro de $A$. serpentina e do parasitoide $A$. anastrephae no estado do Acre. Adicionalmente, Pouteria caimito (Ruiz \& Pav.) Radlk. (Sapotaceae) constitui novo registro de hospedeiro para C. capitata na Amazônia brasileira. Até então, cinco espécies hospedeiras estavam reportadas na região (ADAIME et al., 2017b). Todas as demais associações entre moscas frugívoras e hospedeiros (Tabelas 1 e 2) já haviam sido reportadas na Amazônia brasileira (ADAIME et al., 2016; PEREIRA; ADAIME, 2016) e em outras regiões do Brasil (ZUCCHI, 2008; 2012).

Em Rio Branco, A. striata foi a espécie predominante em função de sua associação com Psidium guajava L. (Myrtaceae), pois infestou todas as três amostras coletadas (Tabela 1). Essa forte associação entre A. striata e $P$. guajava já foi bem documentada na Amazônia brasileira (SILVA et al., 2011a). Do total de moscas frugívoras obtidas (111 espécimes), 55,0\% pertenciam a Anastrepha spp. e 45,0\% a Neosilba spp. Anastrepha obliqua ocorreu em S. mombin e Anacardium occidentale L., ambas Anacardiaceae. Anastrepha striata ocorreu somente em P. guajava. Neosilba glaberrima e N. zadolicha ocorreram apenas em Citrus sinensis (L.) Osbeck (Rutaceae).

Em Senador Guiomard, predominou A. obliqua, em decorrência do número expressivo de espécimes obtidos da amostra de Eugenia stipitata McVaugh (Myrtaceae). Essa associação já foi relatada em outros locais da Amazônia brasileira (ADAIME et al., 2016; SOUZA-ADAIME et al., 2017). Do total de moscas frugívoras obtidas (163 espécimes), 90,2\% pertenciam a Anastrepha spp., 6,1\% a C. capitata e 3,7\% a Neosilba spp. Anastrepha obliqua e N. zadolicha ocorreram em E. stipitata. Anastrepha leptozona, A. serpentina, C. capitata e $N$. glaberrima ocorreram em $P$. caimito. Anastrepha striata ocorreu em P. guajava (Tabela 2).

Os maiores índices de infestação foram obtidos em P. guajava (76,7 pupários/kg) e S.mombin $(62,9$ 
pupários/kg), em Rio Branco (Tabela 1), e em P. guajava (100 pupários/kg) e E. stipitata (96,3 pupários/kg), em Senador Guiomard (Tabela 2). Altas infestações em goiaba (até 263,6 pupários $/ \mathrm{kg}$ ) e $S$. mombin (até 195,0 pupários $/ \mathrm{kg}$ ) também já foram obtidas no estado do Acre (THOMAZINI; ALBUQUERQUE, 2009; PEREIRA et al., 2010). Já a infestação registrada em E. stipitata foi superior à observada por Pereira et al. (2010).

Após este trabalho, estão reportadas oito espécies de Tephritidae (sete espécies de Anastrepha, além de $C$. capitata) e duas de Lonchaeidae para o estado do Acre (ADAIME et al., 2016). Em sete dos 22 municípios do Acre, pelo menos uma espécie de mosca frugívora está relatada. Os municípios com maior número de espécies registradas são Rio Branco e Senador Guiomard, com oito e sete espécies, respectivamente. No entanto, apenas municípios localizados na porção leste do estado foram contemplados com levantamentos, ainda que pontuais.

Chama a atenção o fato de termos obtido somente dois espécimes de parasitoide, especificamente $A$. anastrephae, de amostras de $S$. mombin infestadas por A. obliqua (Tabela 1). Essa espécie já foi obtida de frutos de $S$. mombin em outros estados da Amazônia brasileira (Amapá, Amazonas e Pará) (SOUSA et al., 2016). No entanto, as espécies mais abundantes na região são $D$. areolatus e O. bellus (DEUS; ADAIME, 2013; SOUSA et al., 2016). Além disso, o percentual de parasitismo foi baixo, apenas 2,3\% (dois parasitoides obtidos de 88 pupários), o que provavelmente está relacionado ao baixo índice de emergência $(9,1 \%)$ registrado na amostra (seis espécimes de A. obliqua e duas de A. anastrephae). Thomazini e Albuquerque (2009) registraram parasitismo mais elevado $(29,5 \%)$ a partir de frutos de $S$. mombin coletados no município Bujari.

Anastrepha striata e A. obliqua são as espécies de moscas frugívoras de importância econômica mais abundantes nos municípios amostrados e infestam, respectivamente, P. guajava e E. stipitata. Este trabalho constitui o primeiro registro de $A$. serpentina e do parasitoide $A$. anastrephae para o Acre.

Considerando-se que o conhecimento sobre distribuição, plantas hospedeiras e parasitoides de moscas frugívoras no estado do Acre é bastante escasso, são necessários mais estudos baseados na amostragem de frutos potencialmente hospedeiros. Se possível, sugere-se a separação das amostras de frutos coletados diretamente da copa da planta das amostras de frutos coletados do solo (recém-caídos), pois o índice de infestação e o parasitismo podem ser diferentes em cada situação, como detalhado por Silva et al. (2011a; 2011b).

\section{Agradecimentos}

Ao Dr. Pedro Carlos Strikis pela identificação dos Lonchaeidae. Ao Conselho Nacional de Desenvolvimento Científico e Tecnológico - CNPq, pela Bolsa de Produtividade em Pesquisa concedida a Ricardo Adaime.

\section{Referências}

ADAIME, R.; SANTOS, R. S.;AZEVEDO, T. S.; VASCONCELOS, A. S.; SOUSA, M. S. M.; SOUZA-FILHO, M. F. First record of Ceratitis capitata (Wiedemann) (Diptera: Tephritidae) in the state of Acre, Brazil. EntomoBrasilis, Vassouras, v. 10, n. 3, p. 259-260, $2017 \mathrm{~b}$.

ADAIME, R.; SOUSA, M. S. M.; JESUS-BARROS, C. R.; DEUS, E. G.; PEREIRA, J. F.; STRIKIS, P. C.; SOUZA-FILHO, M. F. Frugivorous flies (Diptera: Tephritidae, Lonchaeidae), their host plants, and associated parasitoids in the extreme north of Amapá State, Brazil. The Florida Entomologist, Lutz, v. 100, n. 2, p. 316324, 2017a.

ADAIME, R.; SOUSA, M. S. M.; PEREIRA, J. F. Anastrepha species and their host in the Brazilian Amazon. 2016. Disponível em: <http://anastrepha.cpafap.embrapa.br $>$. Acesso em: 30 jan. 2018 .

ALUJA, M. Bionomics and management of Anastrepha. Annual Review of Entomology, Palo Alto, v. 39, n. 1, p. 155-178, 1994.

CANAL, N. A.; ZUCCHI, R. A. Parasitoides - Braconidae. In: MALAVASI, A.; ZUCCHI, R. A. (Ed.). Moscas-das-frutas de importância econômica no Brasil: conhecimento básico e aplicado. Ribeirão Preto: Holos, 2000. p. 119-126.

DEUS, E. G.; ADAIME, R. Dez anos de pesquisas sobre moscasdas-frutas (Diptera: Tephritidae) no estado do Amapá: avanços obtidos e desafios futuros. Biota Amazônia, Amapá, v. 3, n. 3, p. $157-168,2013$.

LEMOS, L. N.; PEREIRA, J. D. B.; SILVA, R. A.; MARSARO JUNIOR, A. L. Vulnerabilidade da faixa de fronteira do Brasil à introdução de espécies invasoras exóticas. In: PORTO, J. L. R.; SOTTA, E. D. (Org.). Reformatações fronteiriças no Platô das Guianas: (re)territorialidades de cooperações em construções. Rio de Janeiro: Publit, 2011. p. 121-144.

MARINHO, C. F.; SILVA, R. A.; ZUCCHI, R. A. Chave de identificação de Braconidae (Alysiinae e Opiinae) parasitoides de larvas frugívoras na região Amazônica. In: SILVA, R. A.; LEMOS, W. P.; ZUCCHI, R. A. (Ed.). Moscas-das-frutas na Amazônia 
brasileira: diversidade, hospedeiros e inimigos naturais. Amapá: Embrapa Amapá, 2011. p. 91-101.

McAlPINE, J. F.; STEYSKAL, G. C. A revision of Neosilba McAlpine with a key to the world genera of Lonchaeidae (Diptera). The Canadian Entomologist, Ottawa. v. 114, n. 2, p. 105-137, 1982.

PEREIRA, J. D. B.; BURITI, D. P.; LEMOS, W. P.; SILVA, W. R.; SILVA, R. A. Espécies de Anastrepha Schiner (Diptera: Tephritidae), seus hospedeiros e parasitóides nos estados do Acre e Rondônia, Brasil. Biota Neotropica, Campinas, v. 10, n. 3, p. 441-446, 2010.

PEREIRA, J. D. B.; SILVA, R. A.; LEMOS, W. P. Conhecimento sobre moscas-das-frutas no estado do Acre. In: SILVA, R. A.; LEMOS, W. P.; ZUCCHI, R. A. (Ed.). Moscas-das-frutas na Amazônia brasileira: diversidade, hospedeiros e inimigos naturais. Amapá: Embrapa Amapá, 2011. p. 217-222.

PEREIRA, J. F.; ADAIME, R. Lonchaeidae from Brazilian Amazon. 2016. Disponível em: <http://lonchaeidae.cpafap. embrapa.br>. Acesso em: 31 jan. 2018.

PORTAL BRASIL. Estados brasileiros - Acre. Disponível em $<$ http://www.portalbrasil.net/estados_ac.htm>. Acesso em: 31 jan. 2018.

SILVA, R. A.; DEUS, E. G.; RAGA, A.; PEREIRA, J. D. B.; SOUZA-FILHO, M. F.; COSTA NETO, S. V. Monitoramento de moscas-das-frutas na Amazônia: amostragem de frutos e uso de armadilhas. In: SILVA, R. A.; LEMOS, W. P.; ZUCCHI, R. A. (Ed.). Moscas-das-frutas na Amazônia brasileira: diversidade, hospedeiros e inimigos naturais. Amapá: Embrapa Amapá, 2011b. p. 33-50.

SILVA, R. A.; LEMOS, W. P.; ZUCCHI, R. A. Moscas-das-frutas na Amazônia brasileira: diversidade, hospedeiros e inimigos naturais. Amapá: Embrapa Amapá, 2011a. 299 p.

SOUSA, M. S. M.; ADAIME, R.; PEREIRA, J. F. Fruit flies parasitoids in the Brazilian Amazon. 2016. Disponível em: $<$ http://parasitoid.cpafap.embrapa.br>. Acesso em: 31 jan. 2018.
SOUZA-ADAIME, M. S. M.; JESUS-BARROS, C. R.; SOUSA, M. S. M.; DEUS, E. G.; STRIKIS, P. C.; ADAIME, R. Eugenia stipitata McVaugh (Myrtaceae): food resource for frugivorous flies in the state of Amapá, Brazil. Biotemas, Florianópolis, v. 30, n. 4, p. 129-133, 2017.

STRIKIS, P. C.; DEUS, E. G.; SILVA, R. A.; PEREIRA, J. D. B.; JESUS, C. R.; MARSARO JÚNIOR, A. L. Conhecimento sobre Lonchaeidae na Amazônia brasileira. In: SILVA, R. A.; LEMOS, W. P.; ZUCCHI, R. A. (Ed.). Moscas-das-frutas na Amazônia brasileira: diversidade, hospedeiros e inimigos naturais. Amapá: Embrapa Amapá, 2011. p. 205-216.

THOMAZINI, M. J.; ALBUQUERQUE, E. S. Parasitóides (Hymenoptera: Braconidae) de Anastrepha Schiner (Diptera: Tephritidae) no estado do Acre. Acta Amazônica, Manaus, v. 39, n. 1, p. 245-247, 2009.

THOMAZINI, M. J.; ALBUQUERQUE, E. S.; SOUZA-FILHO, M. F. Primeiro registro de espécies de Anastrepha (Diptera: Tephritidae) no estado do Acre. Neotropical Entomology, Campinas, v. 32, n. 4, p. 723-724, 2003.

THOMAZINI, M. J.; THOMAZINI, A. P. B. W.; SOUZA-FILHO, M. F. Diversidade de moscas-das-frutas (Diptera: Tephritidae) capturas em armadilhas no estado do Acre. In: CONGRESSO BRASILEIRO DE ENTOMOLOGIA, 21, 2006, Recife. Resumos... Recife: UFRPE/SEB, 2006. Versão eletrônica.

ZUCCHI, R. A. Fruit flies in Brazil - Anastrepha species their host plants and parasitoids. 2008. Disponível em: <http: www.lea. esalq.usp.br/anastrepha/>. Acesso em: 12 jun. 2018.

ZUCCHI, R. A. Fruit flies in Brazil - Hosts and parasitoids of the Mediterranean fruit fly. 2012. Disponível em: <http: www.lea. esalq.usp.br/ceratitis/>. Acesso em: 12 jun. 2018.

ZUCCHI, R. A.; URAMOTO, K.; SOUZA-FILHO, M. F. Chave ilustrada para as espécies de Anastrepha da região Amazônica. In: SILVA, R. A.; LEMOS, W. P.; ZUCCHI, R. A. (Ed.). Moscasdas-frutas na Amazônia brasileira: diversidade, hospedeiros e inimigos naturais. Amapá: Embrapa Amapá, 2011. p. 71-90. 